\title{
PSYCHOMETRIC VALIDATION OF THE BAHASA MALAYSIA VERSION OF THE EORTC QLQ-C30 IN MALAYSIAN COLORECTAL CANCER PATIENTS
}

\author{
Bello Arkilla Magaji ${ }^{1}$, 3, 4* Foong Ming Moy ${ }^{1}$, Chee Wei Law ${ }^{2}$ and Ismail Sagap ${ }^{5}$ and April Camilla \\ Roslani ${ }^{2,3}$ \\ ${ }^{1}$ Julius Centre University of Malaya, Department of Social \& Preventive Medicine, Faculty of Medicine, University \\ of Malaya, 50603, Kuala Lumpur, Malaysia \\ ${ }^{2}$ Department of Surgery, Faculty of Medicine, University of Malaya, 50603, Kuala Lumpur, Malaysia \\ ${ }^{3}$ University of Malaya Cancer Research Institute (UMCRI), University of Malaya, 50603, Kuala Lumpur, Malaysia \\ ${ }^{4}$ Department of Community Health, College of Health Sciences, Usmanu Danfodiyo University, PMB 2346, Sokoto, \\ Nigeria \\ ${ }^{5}$ Department of Surgery, Universiti Kebangsaan Malaysia Medical Centre, Jalan Ya'acob Latif, Bandar Tun Razak, \\ Cheras 56000 Kuala Lumpur
}

Corresponding author: Bello Arkilla Magaji

Email(s): $\underline{\text { mbarkilla@gmail.com }}$

\begin{abstract}
This study aimed to assess the validity and reliability of the Bahasa Malaysia (BM) version of European Organization for Research and Treatment of Cancer (EORTC) Quality of Life Questionnaire core (QLQ-C30) (version 3.0) in Malaysian patients with colorectal cancer. A cross sectional study design was used to obtain data from patients receiving treatment at two teaching hospitals in Kuala Lumpur, Malaysia. Self-administered method was used. Statistical analysis included reliability, convergent and discriminant validity and known-groups comparisons. Statistical significance was based on $p$ value $\leq 0.05$. The internal consistency Cronbach's alpha coefficient (a) was acceptable $(>0.70)$ in all scales but cognitive $(a=0.56)$ and pain in patients with stoma bag $(a=0.35)$. Testretest coefficients were high $(r=0.93$ to 1.00). All items showed adequate convergent validity $(r>0.40)$ except for questionnaire item 5 "needs help in eating/dressing/washing". Similarly, criteria for discriminant validity were achieved in all but item 10 "need rest". Patients with high Karnofsky Performance Scores (KPS) scores reported significantly less dyspnoea $(p=0.021)$ and appetite loss $(p=0.047)$ compared to patients with low KPS scores. There was no significant difference between patients with and without stomas. The psychometric properties of the BM version of the QLQ-C3O were comparable to previous studies in other settings. Therefore, the questionnaire could be used to measure quality of life in Malaysian patients with colorectal cancer.
\end{abstract}

Key words: EORTC QLQ-C30-colorectal carcinoma-Malaysia

\section{INTRODUCTION}

The European Organization for Research Treatment of Cancer (EORTC) Quality of Life core (QLQ-C30) questionnaire is designed to be applicable in all cancer patients ${ }^{1}$. Thus, since its development and release in 1993, EORTC QLQ-C30 has been translated, and its' psychometric properties studied, across several settings and cancer types ${ }^{2-10}$. In Malaysia however, the psychometric properties of the QLQ-C30 has not been tested among colorectal cancer (CRC) patients despite the fact that colorectal cancer is the second leading cancer among the general population in Malaysia ${ }^{11}$. In addition, colorectal cancer accounts for $12.7 \%$ of all cancers diagnosed in Malaysia, comprising $15.7 \%$ and $10.4 \%$ of male and female cancers respectively. Nearly equal numbers of colon and rectal cancers were diagnosed $(4,547 ; 15.7$ per 100,000 versus 4,$689 ; 16.2$ per 100,000$)$ with similar mortality rates $(241 ; 0.83$ per $100,000$ versus $229 ; 0.79$ per 100,000$){ }^{11}$. Therefore, evaluating the psychometric properties in Malaysian patients with colorectal cancer is both necessary and timely.

This study aimed to assess the psychometric properties (validity and reliability) of the Bahasa Malaysia (BM) version of EORTC-QLQC30 (version 3.0) in patients with colorectal cancer. This is part of a large project on epidemiology, survival, prognostic factors and health-related quality of life (HRQoL) of Malaysian colorectal cancer patients. The protocol as well as earlier findings from the study was published earlier ${ }^{12-14}$. 


\section{METHODS}

Colorectal cancer patients receiving treatment at University Malaya Medical Centre (UMMC) and University Kebangsaan Malaysia Medical Centre (UKMMC) were included. This study received ethics approval from the Medical Research Ethics Committees of UMMC (PPUM/UPP/300/02/02, MEC 770.2) and UKMMC (Project code: FF-274-2011). Recruitment took place between February 2012 and June 2012. Demographic and clinical data was retrieved from patients' medical records using a predesigned form. Quality of life data was obtained using the Bahasa Malaysia translated version of the EORTC QLQ-C30. The original questionnaire was obtained from the Quality of Life department of EORTC. All participants provide written informed consent. Participants were requested to complete the questionnaire by themselves while waiting to see the doctor at the outpatient surgical clinic or immediately after the consultation. In addition, surgeons rated the patient's overall wellbeing using the Karnofsky Performance Scale (KPS). For the test-retest analysis, thirty patients were requested to complete and return another set of the questionnaire one to two weeks after the first assessment.

Descriptive analysis was performed; continuous data was summarized using mean and standard deviations, while categorical data was summarized using proportions. The internal consistency of the multi-item scales was examined by the use of Cronbach's alpha coefficient. A coefficient of $\geq 0.70$ is considered acceptable. Inter-item correlation coefficient was used to examine test-retest reliability. Multi-trait scaling analyses were used to examine the scale structures (convergent and discriminant validity). A criterion considered is that each item's own scale correlation should exceed 0.4 for convergent validity to be achieved. The discriminant validity measures item correlation with other scales. It is hypothesized that selfcorrelation of an item should be higher than with the other scales. Validity between clinically distinct groups was examined by comparing the scores of patients with and without stomas. In addition, patients were grouped into two; KPS score of $<80 \%$, and $>80 \%$. ) were also compared using Mann Whitney U- tests. All analyses were done via SPSS version 21.0 for Windows, (SPSS Inc., and Chicago, Illinois, USA). A two-tailed probability value of 0.05 was used to determine the level of significance. Further details can be obtained in the published protocol ${ }^{12}$.

\section{RESULTS}

\section{Patient characteristics}

A total of 93 patients (UMMC 47, UKMMC 46) completed the questionnaire. The mean age and standard deviation (SD) was 57 years (SD 11) (range 25 to 74). There were more males (59\%); $28 \%$ were not formally educated. Full time workers accounted for $45 \%$ of the respondents. Stage information was missing for 10 patients. Dukes' stages C and D taken together accounted for $61 \%$ of total patients. The mean KPS was $80 \%$ (SD 10) and $32 \%$ of patients had a stoma (Table 1 ).

\section{Internal consistency and test-retest coefficients}

With regards to the internal consistencies of the GHS/QOL, most of the functioning and multi-symptom scales were acceptable with Cronbach's alpha $\geq 0.70$. The exception was the cognitive functioning scale, which was persistently low across all levels (highest Cronbach's a ranges from 0.51 to 0.56 ); the pain scale was moderate overall (Cronbach's a $=0.63$ ), but low in patients with stoma bag (Cronbach's $\alpha=0.35)$.

The test-retest reliability in the GHS/QOL, functioning scales and in most of the symptoms scales was high ( $r=0.93$ to 1.00 ). The lowest test-retest reliability coefficients were found for appetite loss scale $(r=0.14)$ and dyspnoea $(r=0.53)$.

Multi-trait scaling analyses for convergent and discriminant validity

The convergent $(r \geq 0.40)$ and discriminant validities were achieved in all but question number 5 . The correlation of question number 5 with its proposed domain (the physical functioning scale) was $r=0.38$, which was less than the minimum required coefficient of $r \geq$ 0.40 . On the other hand, this item's correlation with its domain was less than its correlation with other scales such as cognitive $(r=0.43)$ and social/family functioning $(r=0.41)$. 
Table 1: Socio-demographic characteristics of the patients

\begin{tabular}{|c|c|c|c|}
\hline Characteristics & Number (\%) & Employment status & \\
\hline Hospital & & Full time & $42(45)$ \\
\hline UMMC & $46(49)$ & Retired & $36(39)$ \\
\hline UKMMC & $47(51)$ & Unemployed & $7(8)$ \\
\hline Age (years) & & Unknown & $8(9)$ \\
\hline Mean $(S D)$ & $57[11]$ & Site & \\
\hline Range & $25-74$ & Colon & $48(52)$ \\
\hline Gender & & Rectum & $35(38)$ \\
\hline Male & $55(59)$ & Unknown & $10(10)$ \\
\hline Female & $38(41)$ & Dukes' stage & \\
\hline Ethnicity & & $A$ & $4(4)$ \\
\hline Malay & $71(76)$ & B & $26(28)$ \\
\hline Indians & $18(20)$ & C & $17(18)$ \\
\hline Others & $4(4)$ & D & $40(43)$ \\
\hline Educational status & & Unknown & $6(7)$ \\
\hline Primary & $17(18)$ & Stoma & \\
\hline Secondary & $24(26)$ & Yes & $32(34)$ \\
\hline Tertiary & $12(13)$ & No & $61(66)$ \\
\hline Not formally educated & $26(28)$ & $\begin{array}{l}\text { Karnofsky performance status } \\
\text { Mean (SD) }\end{array}$ & $80(10)$ \\
\hline Unknown & $14(15)$ & $\begin{array}{l}\leq 80 \\
\geq 81\end{array}$ & $\begin{array}{l}50(54) \\
43(46)\end{array}$ \\
\hline
\end{tabular}

Table 2: Internal consistency coefficient (Bahasa Malaysia)

\begin{tabular}{lccc}
\hline \multicolumn{1}{c}{ Scale } & \multicolumn{3}{c}{ Cronbach's alpha } \\
\cline { 2 - 4 } & Overall & With stoma & Without stoma \\
\hline Global health status/quality of life & & & \\
$\quad$ Global health status/quality of life & 0.9 & 0.93 & 0.87 \\
Functioning scales & & & 0.76 \\
$\quad$ Physical & 0.75 & 0.75 & 0.89 \\
Role & 0.91 & 0.93 & 0.85 \\
Emotional & 0.86 & 0.87 & $0.51^{*}$ \\
Cognitive & $0.53^{*}$ & $0.56^{*}$ & 0.92 \\
Social and family & 0.84 & 0.74 & 0.7 \\
Multi-item symptoms scales & & & 0.71 \\
Fatigue & 0.7 & 0.7 & 0.73 \\
Nausea/Vomiting & 0.77 & 0.83 & \\
Pain & $0.64^{*}$ & $0.35^{*}$ & \\
\hline
\end{tabular}


Table 3: Multi-trait scaling analysis (Bahasa Malaysia)

\begin{tabular}{|c|c|c|c|c|c|c|c|c|c|c|}
\hline \multirow{2}{*}{\multicolumn{2}{|c|}{ Questionnaire item }} & \multirow{2}{*}{ GHS/QOL } & \multicolumn{5}{|c|}{ Functioning scales } & \multicolumn{3}{|c|}{ Multi-item symptom scales } \\
\hline & & & Physical & Role & Cognitive & Emotional & $\begin{array}{l}\text { Social } \\
\text { Family }\end{array}$ & Fatigue & $\begin{array}{l}\text { Nausea/ } \\
\text { Vomiting }\end{array}$ & Pain \\
\hline \multicolumn{11}{|c|}{ GHS/QOL } \\
\hline 29 & Global Health Status & 0.95 & 0.33 & 0.30 & 0.36 & 0.41 & 0.29 & -0.42 & -0.31 & -0.44 \\
\hline 30 & Quality of Life & 0.96 & 0.37 & 0.41 & 0.40 & 0.49 & 0.34 & -0.51 & -0.29 & -0.48 \\
\hline \multicolumn{11}{|c|}{ Functioning scales } \\
\hline \multicolumn{11}{|c|}{ Physical } \\
\hline 1 & Strenuous activity & -0.39 & -0.79 & -0.60 & -0.33 & -0.36 & -0.38 & 0.46 & 0.29 & 0.57 \\
\hline 2 & Long walk & -0.32 & -0.82 & -0.49 & -0.34 & -0.36 & -0.33 & 0.33 & 0.24 & 0.63 \\
\hline 3 & Short walk & -0.27 & -0.79 & -0.33 & -0.26 & -0.19 & -0.25 & 0.23 & 0.16 & 0.38 \\
\hline 4 & Stay in bed/chair & -0.12 & -0.68 & -0.26 & -0.27 & -0.25 & -0.25 & 0.29 & 0.15 & 0.28 \\
\hline 5 & $\begin{array}{l}\text { Need help in } \\
\text { eating/dressing/ } \\
\text { washing }\end{array}$ & -0.16 & $-0.38^{*}$ & -0.18 & $-0.43^{*}$ & -0.23 & $-0.41^{*}$ & 0.08 & 0.33 & 0.19 \\
\hline
\end{tabular}

GHS/QOL, global health status/quality of life

*Question number 5 correlations with physical, cognitive and social/family functioning scales 
Table 3, continued

\begin{tabular}{|c|c|c|c|c|c|c|c|c|c|c|}
\hline \multirow{2}{*}{\multicolumn{2}{|c|}{ Questionnaire item }} & \multirow[t]{2}{*}{ GHS/QOL } & \multicolumn{5}{|c|}{ Functioning scales } & \multicolumn{3}{|c|}{ Multi-item symptom scales } \\
\hline & & & \multirow[t]{2}{*}{ Physical } & \multirow[t]{2}{*}{ Role } & \multirow[t]{2}{*}{ Cognitive } & \multirow[t]{2}{*}{ Emotional } & \multirow[t]{2}{*}{$\begin{array}{l}\text { Social } \\
\text { Family }\end{array}$} & \multirow[t]{2}{*}{ Fatigue } & \multirow[t]{2}{*}{$\begin{array}{l}\text { Nausea/ } \\
\text { Vomiting }\end{array}$} & \multirow[t]{2}{*}{ Pain } \\
\hline Role & & & & & & & & & & \\
\hline 6 & Limited work & -0.34 & -0.54 & -0.95 & -0.34 & -0.37 & -0.45 & 0.48 & 0.28 & 0.65 \\
\hline 7 & Limited hobbies & -0.38 & -0.52 & -0.96 & -0.39 & -0.35 & -0.51 & 0.51 & 0.34 & 0.62 \\
\hline \multicolumn{11}{|c|}{ Cognitive } \\
\hline 20 & Concentration & -0.31 & -0.35 & -0.44 & -0.81 & -0.35 & -0.38 & 0.39 & 0.49 & 0.47 \\
\hline 25 & Memory & -0.35 & -0.36 & -0.20 & -0.84 & -0.47 & -0.36 & 0.39 & 0.35 & 0.35 \\
\hline \multicolumn{11}{|c|}{ Emotional } \\
\hline 21 & Tense & -0.50 & -0.39 & -0.32 & -0.41 & -0.83 & -0.52 & 0.36 & 0.30 & 0.53 \\
\hline 22 & Worried & -0.39 & -0.32 & -0.31 & -0.41 & -0.86 & -0.45 & 0.49 & 0.19 & 0.48 \\
\hline 23 & Irritable & -0.25 & -0.15 & -0.14 & -0.32 & -0.75 & -0.29 & 0.24 & 0.10 & 0.27 \\
\hline 24 & Depressed & -0.42 & -0.43 & -0.35 & -0.52 & -0.89 & -0.45 & 0.56 & 0.33 & 0.48 \\
\hline \multicolumn{11}{|c|}{ Social/Family } \\
\hline 26 & Family life & -.035 & -0.23 & -0.40 & -0.38 & -0.42 & -0.92 & 0.31 & 0.21 & 0.47 \\
\hline 27 & Social life & 0.01 & -0.47 & -0.52 & -0.45 & -0.53 & -0.94 & 0.48 & 0.43 & 0.49 \\
\hline
\end{tabular}

GHS/QOL: global Health Status/Quality of Life 
Clinically distinct group comparisons

We observed a trend where patients with a stoma bag reported lower mean scores for $\mathrm{GHS} / \mathrm{QOL}$, and functioning scores, as well as more symptoms compared to patients without a stoma. However, the differences were not statistically significant. With reference to KPS scores, patients with high KPS scores reported significantly less dyspnoea $(p=0.021)$ and appetite loss $(p=0.047)$ compared to patients with low KPS scores.

Table 4: Group comparison according to Karnofsky Performance Scores (KPS) for EORTC QLQ-C30 versions

\begin{tabular}{|c|c|c|c|}
\hline \multirow[t]{4}{*}{ Domains } & \multicolumn{2}{|c|}{ Bahasa Malaysia } & \multirow[t]{4}{*}{$\mathrm{p}$-value } \\
\hline & $\leq 80$ & $\geq 81$ & \\
\hline & $(n=50)$ & $(n=43)$ & \\
\hline & Mean (SD) & Mean (SD) & \\
\hline \multicolumn{4}{|l|}{ GHS/QOL } \\
\hline GHS/QOL & $70.33(23.52)$ & $74.81(14.14)$ & 0.063 \\
\hline \multicolumn{4}{|l|}{ Functioning scales } \\
\hline Physical & $72.27(23.30)$ & $82.48(15.67)$ & 0.055 \\
\hline Role & $67.67(38.59)$ & $76.36(25.26)$ & 0.755 \\
\hline Emotional & $75.83(23.59)$ & $81.20(20.66)$ & 0.291 \\
\hline Cognitive & $84.67(17.44)$ & $82.94(17.63)$ & 0.535 \\
\hline Social/Family & $82.00(22.55)$ & $83.72(21.35)$ & 0.759 \\
\hline \multicolumn{4}{|c|}{ Multi-items symptom scales } \\
\hline Fatigue & $40.22(25.18)$ & $30.23(17.87)$ & 0.063 \\
\hline Nausea/Vomiting & $11.00(17.69)$ & $6.59(17.49)$ & 0.054 \\
\hline Pain & $29.67(26.99)$ & $20.54(20.19)$ & 0.121 \\
\hline \multicolumn{4}{|l|}{ Single item/symptoms } \\
\hline Dyspnoea & $18.67(25.34)$ & $7.75(17.57)$ & $0.021^{*}$ \\
\hline Sleep loss & 28.67(31.59) & $24.03(25.53)$ & 0.646 \\
\hline Appetite loss & $25.33(33.37)$ & $11.63(21.68)$ & $0.047^{*}$ \\
\hline Constipation & $20.00(34.99)$ & $17.83(24.50)$ & 0.628 \\
\hline Diarrhoea & $12.00(24.05)$ & $17.05(25.58)$ & 0.207 \\
\hline Financial difficulty & $26.00(35.82)$ & $20.15(27.35)$ & 0.695 \\
\hline
\end{tabular}

*Significant difference $(\mathrm{p}<0.05)$, GHS/QOL: Global Health Status/Quality of Life 


\section{DISCUSSION}

Evaluation of the reliability and validity of the Bahasa Malaysia version of the EORTC QLQ-C30 was both necessary and timely. The psychometric properties obtained in this study were comparable to the previous studies ${ }^{15}$. The persistent poor internal consistency coefficients for cognitive function scale were observed in previous validation studies of the QLQ-C30 in Indonesia ${ }^{9}$, Singapore ${ }^{10}$, Korea ${ }^{6}$, simplified Chinese ${ }^{3}$, and Malay patients with breast cancer $^{7}$. In these studies, the alpha ranges from 0.19 to 0.67 . However, the poor internal consistency pain scale obtained in patients with stomas contradicts previous studies $^{3,9,10}$. Appetite loss low test-retest correlation is likely due to transient nature of the appetite loss symptom, especially if it was not as a result of any organic cause.

With reference to the convergent-discriminant validity, all the items in the questionnaire met the criteria for the convergent and discriminant validity with exception of item 5 "need help in eating/dressing/washing". We found the questionnaire item correlation with the social and family function scale was higher than its correlation with the proposed physical functioning scale. The study findings were similar to the Indonesian and several other studies where questionnaire item number 5 correlate higher with emotional function than its own proposed physical function scale ${ }^{3,9,16}$.

Our findings on clinical utility of the questionnaire showed that appetite loss was the only domain that differentiated between patients with and without stoma bag and that dyspnoea and appetite loss demonstrated significant difference between patients in two spectra of the Karnofsky performance scale. A recent Cochrane review found that variations in HRQOL domains between patients with and without stoma were not consistent ${ }^{17}$. Several previous studies failed to identify differences between patients with different cancers stages $^{9}$, and co-morbid conditions ${ }^{10}$. However, patients with the worse ECOG performance scores reported poor functioning, global health status/Quality of life, and higher symptoms compared to those with good/higher ECOG ${ }^{18}$. Our study is the first to attempt to examine the psychometric properties of the Malaysian versions of the EORTC QLQ- QLQ-C30 among Malaysian colorectal cancer patients. The limitation of this study is the use of patients from urban setting only. Further studies might be needed to compare our findings with that of patients from rural setting.

\section{CONCLUSION}

Our study findings indicated that the Bahasa Malaysia version of the QLQ-C30 is a valid and reliable measure of $\mathrm{HRQOL}$ in patients with colorectal cancer. This demonstrated the global applicability of the QLQ-C30 in the assessment of cancer patients' HRQOL. Clinicians should make appropriate use of these tools and examine the possibility of collecting HRQOL as part of the routine clinical follow up of care. This could be done before and after commencement of major treatment modalities such as surgery, chemotherapy or radiotherapy.

\section{ACKNOWLEDGEMENTS}

This study was fully funded by the University of Malaya postgraduate fund with the following reference numbers (UM-IPPP PS212/2010A and PV015-2011B), and partially supported by the STeMM Programme, the University of Malaya / Ministry of Higher Education (UM/MOHE) High Impact Research Grant (No: E000010-20001). The authors thank the patients, nurses and members of the colorectal and oncology units of the UMMC and HUKM for the assistance they rendered towards the success ofthis study. We thank DagmaraKulis, Maria and other staff of the European Organizationfor Research and Treatment of Cancer Quality of LifeGroup for their extensive support offered to us the translated versions of the $\mathrm{C} 30$.

\section{COMPETING INTEREST}

None declared.

\section{REFERENCES}

1. Luckett $T^{1}$, King MT, Butow PN, et al. Choosing between the EORTC QLQ-C30 and FACT-G for measuring healthrelated quality of life in cancer clinical research: issues, evidence and recommendations. Ann Onco. 2011; 22(10): 2179-90.

2. Arraras Urdaniz JI, Villafranca Iturre E, Arias de la Vega $F$, et al. The eortc quality of life questionnaire QLQ-C30 (version 3.0). Validation study for Spanish prostate cancer patients. Arch Esp Urol. 2008; 61: 949-54

3. Cheng JX, Liu BL, Zhang X, et al. The validation of the standard chinese 
version of the european organization for research and treatment of cancer quality of life core questionnaire 30 (EORTC QLQ-C30) in pre-operative patients with brain tumor in China. BMC Med Res Methodol. 2011; 11: 56

4. Guzelant A, Goksel T, Ozkok S, et al. The european organization for research and treatment of cancer QLQ-C30: an examination into the cultural validity and reliability of the Turkish version of the EORTC QLQ-C30. Eur J Cancer Care. 2004; 13: 135-44

5. Jayasekara H, Rajapaksa LC, Aaronson NK. Quality of life in cancer patients in South Asia: psychometric properties of the Sinhala version of the EORTC QLQC30 in cancer patients with heterogeneous diagnoses. Qual Life Res. 2008;17(5): 783-91

6. Lee, E. H., Chun, M., Wang, H, et al. Multidimensional constructs of the EORTC Quality of Life Questionnaire (QLQ-C30) in Korean cancer patients with heterogeneous diagnoses. Cancer Res Treat. 2005; 37(3): 148-56.

7. Yusoff N, Low WY, \& Yip CH. The Malay version of the European organization for research and treatment of cancer quality of life questionnaire (EORTCQLQ (30): realibility and validity study. International Medical Journal Malaysia. 2010; 9(2): 45-50.

8. Huang, C. C., Lien, H. H., Sung, Y. C, et al. Quality of life of patients with gastric cancer in Taiwan: validation and clinical application of the Taiwan Chinese version of the EORTC QLQ-C30 and EORTC QLQ-STO22. Psychooncology. 2007; 16(10): 945-9.

9. Perwitasari, D. A., Atthobari, J., Dwiprahasto, I, et al. Translation and Validation of EORTC QLQ-C30 into Indonesian Version for Cancer Patients in Indonesia. Jpn J Clin Oncol. 2011; 41(4): 519-29.

10. Luo, N., Fones, C. S. L., Lim, S. E, et al. The European Organization for Research and Treatment of Cancer Quality of Life Questionnaire (EORTC QLQ-(30): Validation of English version in Singapore. Qual Life Res. 2015; 14(4): 1181-6.

11. Yahaya DD, Yoong CC, Sulaiman DD, et al (2011). Annual report 2008 Kuala Lumpur: Ministry Of Health Malaysia
12. Magaji, B. A., Moy F.M., Law, C.W, et al. Health-related quality of life among colorectal cancer patients in Malaysia: A study protocol. BMC Cancer. 2012; 384.

13. Magaji, B. A., Moy F.M., Roslani A.C, et al. Psychometric Validation of the Malaysian Chinese Version of the EORTC QLQ-C30 in Colorectal Cancer Patients. Asian Pac J Cancer Prev. 2015; 16 (18), 8107-12

14. Magaji, B. A., Moy F.M., Roslani A.C, et al. Psychometric Validation of the Bahasa Malaysia Version of the EORTC QLQ-CR29. Asian Pac J Cancer Prev. 2015; 16 (18), 8101-5.

15. Uwer, L., Rotonda, C., Guillemin, F, et al. Responsiveness of EORTC QLQ-C30, QLQ-CR38 and FACT-C quality of life questionnaires in patients with colorectal cancer. Health Qual Life Outcomes. 2011; 9: 70.

16. Hoopman, R., Muller, M. J., Terwee, C. $B$, et al. Translation and validation of the EORTC QLQ-C30 for use among Turkish and Moroccan ethnic minority cancer patients in the Netherlands. Eur J Cancer.2006; 42(12): 1839-47.

17. Pachler, J., \& Wille-Jorgensen, P. Quality of life after rectal resection for cancer, with or without permanent colostomy. Cochrane Database of Syst Rev. 2012;12: CD004323. 